Andreas Dorn

\title{
Maiherperi: Ein Grab - drei Bücher
}

Orsenigo, Christian: La Tombe de Maiherperi (KV 36). Milano: Libreria Antiquaria Pontremoli 2016. 213 S. m. Abb., 6 Taf. $8^{\circ}=$ EDAL Supplements 1. Brosch. $€ 65,00$. ISBN 978-8-89419-860-7.

Lakomy, Konstantin C.: Der Löwe auf dem Schlachtfeld. Das Grab KV 36 und die Bestattung des Maiherperi im Tal der Könige. Wiesbaden: Reichert 2016. 512 S., 552 Abb. $4^{\circ}$. Hartbd. € 198,00. ISBN 978-3-95490-110-4.

Besprochen von Andreas Dorn: Uppsala / Schweden, E-Mail: andreas.dorn@egyptologi.uu.se

https://doi.org/10.1515/olzg-2020-0001

Im Jahre 2016, mehr als 110 Jahre nach der Erstpublikation des Grabes des Maiherperi im Tal der Könige (KV 36) durch Georges Daressy, ${ }^{1}$ erschienen nahezu zeitgleich zwei ganz unterschiedliche Publikationen zum Grab und der 1899 durch Victor Loret fast intakt vorgefundenen Grabausstattung. Christian Orsenigos „La tombe de Maiherperi (KV 36)“ stellt, wie der Autor in der Einleitung darlegt, eine überarbeitete Version eines Teils seiner Dissertation dar. Diese enthielt weitere durch Loret im Tal der Könige entdeckte Gräber (KV 34, KV 35; KV 37; KV 38). Das Hauptaugenmerk der Publikation liegt auf der detailgetreuen Rekonstruktion der Position der Grabausstattungsobjekte im Grab, welche dank der Auswertung der als verschollen gegoltenen und wieder entdeckten Grabungstagebücher Lorets möglich geworden ist. Als Basis hierzu dient der mit kleinen schwarz-weiss Fotos bebilderte Katalog mit allen Angaben zu den Objekten aus den Grabungstagebüchern Lorets, den Angaben im Journal d'Entrée (inkl. Nummern) des Museums in Kairo und den Angaben in der Erstpublikation der Objekte (inkl. ihrer Nummern) durch Daressy im Catalogue Général des Museums Kairo, wobei sich Orsenigo bei den Literaturangaben explizit auf ein Minimum beschränkt.

Bei Konstantin C. Lakomys „Der Löwe auf dem Schlachtfeld”. Das Grab KV 36 und die Bestattung des Maiherperi im Tal der Könige“ handelt es sich ebenfalls um eine Dissertation, die indes einen anderen Ansatz verfolgt.

1 Daressy, G. M., Tombe de Maherpra. Catalogue général des antiquités égyptiennes du Musée du Caire CG 24901-24990. Fouilles de la vallée des rois, Kairo 1902 stellt das erste der drei Bücher dar und wurde durch die beiden hier besprochenen Publikationen umfassend rezipiert.
Lakomys Arbeit besteht aus einer vollständigen Dokumentation der gesamten Grabausstattung basierend auf eigenen Beschreibungen, Fotos und Zeichnungen der Originale und einer umfassenden Verortung der Objekte und des Grabes Maiherperis und setzt sich zum Ziel (S. 14), die „Diskussion um den Bestattungszeitpunkt ... abzuschließen“ und eine möglichst genaue Datierung vorzulegen. Dabei wurde die Publikation im Vergleich zur Dissertation noch erweitert (S. 7). Die akkuraten Beschreibungen und der umfangreiche Tafelteil sind dabei besonders hervorzuheben.

Im Folgenden wird zuerst Orsenigos Publikation vorgestellt, wobei bei identischen Themen in Klammern auf denselben Sachverhalt bei Lakomy verwiesen wird (L: S. XX-XX), um die parallele Nutzung der Publikationen zu erleichtern.

Orsenigo beginnt seine Untersuchung (Kapitel I: S. 19-25) zur Person Maiherperis mit der Feststellung, dass dieser nur durch Aufschriften bezeugt ist, welche auf Objekten seiner Grabausstattung angebracht waren. Zusätzliche historische Quellen zu Maiherperi oder weitere Objekte sind bisher nicht bekannt. Hinweise auf sein Leben liefern seine Titel $\underline{h} r d n k 3 p$ (L.: S. 66-68) und $\underline{t 3 y} h w$ (L.: S. 68-70) sowie Objekte seiner Grabausstattung. Der erste Titel „Kind des Kap“ bezeugt, dass er am Königshof zusammen mit Prinzen aufgewachsen ist, der zweite Titel „Wedelträger“ (in mehreren Varianten), dass er zum engsten Umfeld des Königs gehörte.

Durch seine dunkle Hautfarbe ist er gemäss Orsenigo und Lakomy ethnisch als Nicht-Ägypter identifizierbar. Biographisch lassen sich daraus jedoch gemäss dem Rezensenten keine gesicherten Schlüsse ziehen (anders Orsenigo S. 19 in der Überschrift zu Kap. I „Maherperi: un étranger à la cour du roi“; ähnlich L: S. 61-66), ebenso wenig wie aus seinem Namen „der Löwe (ist) auf dem Schlachtfeld“, außer wenn er diesen Namen nach außergewöhnlichen Taten in einer kriegerischen Auseinandersetzung angenommen hätte, wobei wir mit dieser Annahme bereits bei Spekulationen zu Maiherperis Person und Leben wären.

Auffällig sind die Schreibvarianten (S. 25) zum insgesamt 53 Mal überlieferten Namen Maiherperis (L.: S. 64-65), von denen auf seinen Grabausstattungsobjekten nicht weniger als 24 belegt sind, davon allein 14 (Lakomy nennt 15 basierend auf der Unterscheidung einer 
identischen horizontalen von einer vertikalen Schreibung) in seinem Totenbuchpapyrus.

In Kapitel II (S. 27-39) zeichnet Orsenigo die Entdeckungsgeschichte der einzelnen Objekte anhand der Angaben in den Grabungstagebüchern von Victor Loret nach. Daraus geht klar hervor, welche Objekte von Loret wie bezeichnet wurden, wo sie sich befanden und in welcher Reihenfolge sie geborgen wurden. Durch schematische Zeichnungen der Fundsituation mit daneben positionierten Fotos werden die Tagebucheinträge für den Leser ideal illustriert.

Von besonderem Interesse ist Kapitel III (S. 41-47), in welchem erstmals die Listen publiziert werden, in welchen durch den Ausgräber festgehalten wurde, in welchen Kisten welche Objekte nach Kairo verbracht wurden. Zudem sind die Listen datiert; Liste II trägt das Datum 7. April 1899, woraus ersichtlich wird, dass die Grabausstattung gut eine Woche, nachdem das Grab am 30. März durch Victor Loret entdeckt wurde, bereits reisefertig verpackt bzw. der Versand soweit vorbereitet war.

Kapitel IV (S. 49-147) umfasst den vollständigen Objektkatalog unter Angabe der Nummern des Catalogue Général, des Journal d'Entrée sowie einer eigenen Nummerierung, welche in ihrer Abfolge die Reihenfolge der Objektbezeichnungen durch Loret beim Auffinden der Objekte berücksichtigt. Die meisten Einträge sind durch ein kleines s/w-Foto illustriert, die bibliografischen Angaben kurz gehalten, wobei bei der ganzen Arbeit der Fokus auf der Aufarbeitung der damaligen Prozesse liegt, so dass jeweils die Angaben in BIE (Bulletin de l'Institut Égyptien) und PM (Porter/Moss) systematisch angegeben werden und bei den aktuellen Verweisen nur dann ausführlichere Literaturangaben vermerkt sind, wenn Objekte u. a. für Datierungsfragen des Grabes etc. in der Publikation zusätzlich diskutiert werden. Abgeschlossen wird dieses Kapitel durch eine Konkordanz der Nummern unter Angabe von Fehlbezeichnungen u.a. in der Publikation der Grabausstattung durch Daressy von 1902.

Einige Objekte der Grabausstattung Maiherperis, die Howard Carter 1902 in der Nähe des Grabes gefunden hat, werden in Kapitel V (S. 149-151; L: S. 258-262) vorgestellt. Sie scheinen im Nachgang zur antiken Beraubung des Grabes zurückgelassen worden zu sein (L: 258-262).

Im abschließenden Kapitel VI (S. 153-165) werden Fragen behandelt, die in der Forschung seit der Entdeckung des Grabes kontrovers diskutiert wurden:

- Die Objekte, die auf Grund der Grabberaubung fehlen (S. 153; L.: S. 264-266, deutlich ausführlicher und präziser)

- Die Beraubung (S. 154; L.: S. 266): Roehrig folgend interpretiert Orsenigo die Beraubung als Teil eines offiziellen Vorgangs zur Beschaffung wertvoller Güter, wobei er die Argumentation von Nicholas Reeves und eine Beraubung in der Ramessidenzeit zurückweist. ${ }^{2}$ Lakomy setzt das Beraubungsdatum ans Ende der 21. Dynastie.

Der mögliche Beraubungszeitpunkt lässt unter Einbezug der Graffiti, welche sich an der steil aufragenden, bis zu $8 \mathrm{~m}$ hohen Felswand direkt nördlich des Grabes befinden - an ihrem Fuss wurde durch H. Carter die Holzkiste Maiherperis gefunden - relativ gut eingrenzen, da sich mit Hilfe der Anbringungshöhen der Graffiti die ungefähre Schutthöhe im Bereich vor der Felswand bestimmen lässt. ${ }^{3}$ Bis in die Regierungszeit Ramses II. (Schreiber Qenherkhepeshef) befanden sich keine nennenswerten Schuttmengen vor der Felswand. Durch die Deponierung von Grabaushub der umliegenden Gräber der späten 19. Dynastie stieg das Niveau auf über $5 \mathrm{~m}$ über dem Schacht von Maiherperis Grab an, um zurzeit von Djehutimose und Butehamun am Übergang von der 20. zur 21. Dynastie auf der Suche nach verborgenen Grabanlagen nochmals auf ca. $3 \mathrm{~m}$ abgetieft zu werden. Dieser Befund zeigt, dass die Beraubung vor der 2. Hälfte der 19. Dynastie stattgefunden haben muss und eine Beraubung zeitnah zur Bestattung, wie sie für viele Erstberaubungen angenommen wird, wahrscheinlich wird, ob als offizieller Vorgang oder durch Grabräuber, darüber kann nur spekuliert werden, wobei z. B. das Fehlen des Uschebtis bei solchen Spekulationen einen interessanten Aspekt darstellt.

- Die älteren auf Georg Schweinfurths Beschreibung basierenden Vorschläge zur Position der Grabausstattungsobjekte (S. 154-156)

- Die Datierung des Grabes (S. 157-161), welches Orsenigo, Catharine Roehrig ${ }^{4}$ folgend, um das 20. Jahr der Regierungszeit Thutmosis' III. ansetzt, wobei eine Auflistung zahlreicher Arbeiten, in denen eine jüngere oder auch gleiche Datierung des Grabinventars vertreten wird, es dem Leser erlaubt, sich weiter mit der Datierungsproblematik zu beschäftigen. Es fällt auf, dass Orsenigo keine Keramikdatierungen

2 Reeves, N. C., Valley of the Kings. The decline of a royal necropolis, London 1990, 143 und 146.

3 Siehe hierzu und speziell zu dieser Felswand Sadek, A. A., Varia Graffitica, in: VA 6, 1990, 109-120.

4 Roehrig, C. H., The tomb of Maiherperi in the Valley of the Kings, in: Roehrig C. H., Dreyfus, R., Keller C. A., Hatshepsut. From Queen to Pharaoh, New York 2005, 70-72, "dating between her death and the destruction of her monuments.” 
erwähnt. Roehrigs Ansatz folgt auch Lakomy (s.u.), wobei dieser methodisch problematisch ist:

$\mathrm{Zu}$ Belegen bisheriger Datierungen, die sich zum Beispiel auf das Aufkommen von Ohrringlöchern bei Männern ab der Regierungszeit Amenophis' II. stützen, fügt Roehrig „However, earrings have been found in men's tombs that date from the time of Hatsheput and Thutmose III“5 einige ältere Belege hinzu, ohne weitere Angaben (Besitz des Mannes, Beigabengeschenk, von Mann getragen, weist Mumie Ohrringlöcher auf?) zu machen. Dadurch wird die Aussage, dass Ohrringlöcher an Mumien ab Amenophis II. belegt sind und danach an männlichen Mumien vermehrt auftreten nicht falsifiziert, sondern es wird dadurch nur die Belegspanne erweitert. In der Folge zur Nennung weiterer Belege müsste eine Diskussion erfolgen, in welcher geklärt wird, wie der Beleg Maiherperis innerhalb der neuen Belegspanne anzusetzen ist. Angaben hierzu finden sich bei Roehrig jedoch nicht und so wird suggeriert, dass der Beleg bei Maiherperi automatisch früher ist. Diesem methodisch fragwürdigen Muster scheinen Orsenigo und Laokmy zu folgen.

- Die Interpretation von im Grab Maiherperis (siehe auch KV 46 von Yuia und Tuia) in Gefäßen deponierten Balsamierungsresten, welche Orsenigo mit guten Argumenten als wohl gängige Praxis anspricht (S. 162-165), womit er sich berechtigterweise gegen die These ausspricht, dass es sich beim Grab primär um eine embalming cache gehandelt hätte. ${ }^{6}$

Auf die Bibliographie (S. 169-192) folgen die Indices (S. 195-213) mit jeweils jeder Nummer (Katalognummer KV 36.1 ff., CG-Nummer, JdE-Nummer) in Erstposition, wobei hier Lorets Objektnummern vermisst werden, da diese Nummern im Resultat der Arbeit, dem Plan mit der Position der einzelnen Grabausstattungsobjekte (Taf. VI), zur Objektmarkierung dienten. Abgesehen vom fehlenden Nordpfeil ist es bedauerlich, dass die beiden Seiten, welche die doppelseitige Abbildung bilden, nicht auf gleicher Höhe ins Buch eingebunden wurden und dass Teile der Abbildung in der Buchbindung liegen und deshalb nicht sichtbar sind.

5 Ebenda, 70.

6 Ausführlich bereits Lakomy vor der Publikation des hier besprochenen Werkes „Embalming Chaches“ im Tal der Könige. Eine Bestandsaufnahme für die 18. Dynastie, in: GM 228, 2011, 21-32, spez. $27-28$.
Lakomys Illustration der Fundlage (Plan Abb. 35) deckt sich weitestgehend mit der Rekonstruktion (Taf. VI) bei Orsenigo, obwohl ihm nicht alle Unterlagen, die Orsenigo auswertete, zur Verfügung standen. Als offensichtlichster Unterschied ist einzig die objektfreie Südwestecke (Orsenigo) anzuführen, in welcher Lakomy einige Objekte platzierte.

Lakomys erstes Kapitel (I.1; S. 19-21) beschreibt die Anfänge der Nutzung des Tals der Könige als königlichen Begräbnisplatz ab Thutmosis I. und die pharaonisch belegten Toponyme für das Tal und die Nekropole. Bei der Diskussion topographischer Begebenheiten für die Wahl des Tals als Begräbnisplatz geht Lakomy auch auf die kleinen Schreine/Kapellen ein, die sich am Abhang der Bergspitze Qurn befinden, die das Tal der Könige überragt. Er führt diese kleinen Kapellen unter Verwendung wechselnder teils irreführender Terminologie „Steinbauten en miniature, Steingebilde, Feuersteingebilde, "Feuersteinaltäre”, "Felsheiligtümer”, Miniatur-Sakralbauten“ mit verschiedenen Hypothesen als Argumente gegen den von Adrom $^{7}$ klar herausgearbeiteten Befund an, dass es keine Belege für die Gleichsetzung der Qurn mit der lokalen Göttin Meretseger oder anderen weiblichen Gottheiten gibt (zur Diskussion siehe wiederholt S. 84). Hier fehlt der Verweis auf die Erstpublikation dieser kleinen Schreine von Davies ${ }^{8}$, in welcher einige wenige Ostraka erwähnt sind, die ursprünglich in den kleinen Schreinen aufgestellt waren, wobei Amun und nicht Meretseger auf diesen Ostraka belegt ist, was gegen die Spekulationen von Lakomy spricht. Zudem bleibt anzumerken, dass Meretseger, die nahe gelegene „station de repos“ (Ansammlung von Arbeiterhütten auf halber Strecke zwischen dem Tal der Könige und dem Wohnort der Arbeiter in Deir el-Medine) und die in den kleinen Kapellen gefundenen Ostraka alle erst ab der 19. Dynastie belegt sind und es gerade vor dem Hintergrund, dass in der 19. Dynastie eine Neukonzeption des Königsgrabes (vom verborgenen zum sichtbaren Grabzugang, von teilweise dekoriert zu voll ausdekoriertem Grab etc.) und der Nutzung des Tals der Könige dagegen spricht, Befunde aus der 19. Dynastie zur Erklärung (pragmatisch und religiös) für die 200 Jahre davor, zu Beginn der 18. Dynastie, erfolgte Wahl des Tals der Könige als Begräbnisplatz heranzuziehen. In Kapitel I.2 (S. 21-39) folgt eine Untersuchung von 44 nicht-königlichen und 4 königlichen Bestattungen der Thutmosidenzeit um zu klären, ob anhand ihrer Lage, Architektur, Dekoration und

7 Adrom, F., Der Gipfel der Frömmigkeit? Überlegungen zur Semantik und religiösen Symbolik von $t$ 3-dhn.t, in: LingAeg 12, 1-20.

8 Davies, N. de Garis, A high place at Thebes, in: Anonymous (Hg.), Mélanges Maspero I. Orient Ancien 1, MIFAO 66, Kairo 1934, 241-250. 
„ihrer (noch) vorhandenen Ausstattung“ KV 36 exakter datiert werden kann, wobei die abschließende Datierung von KV 36 in die Zeit Hatschepsuts bzw. Thutmosis' III. (S. 14-15, 21, 81-83) mit Verweis auf den Katalog mit der Analyse des Grabinventars bereits hier vorweggenommen wird. Die Auflistung und Besprechung der Gräber erfolgt anhand der hierfür relevanten älteren Literatur ${ }^{9}$ und wird durch zahlreiche Literaturverweise auf rezentere Projekte bereichert. Sie stellt ein wertvolles Arbeitsinstrument für die Beschäftigung mit diesen Gräbern dar. KV G ist aus der Liste von (begonnenen) Gräbern zu streichen, da es sich bei dieser Struktur um eine senkrechte Abarbeitung des anstehenden Felsens handelt, welche die Rückwand einer Arbeiterhütte bildet. ${ }^{10}$

Irritierend ist die Angabe zur Lage dieser 44 plus 4 Gräber „In der Osthälfte des Tals, ca. 290 m Luftlinie von KV 36 entfernt“" (S. 22ff.) mit Verweis auf den Gesamtplan des Tals der Könige. Für dieses Kapitel wäre ein einmaliger interner Verweis auf Taf. 2, der diesen Plan enthält, benutzerfreundlicher. Durch die Angabe der Entfernung der jeweiligen Gräber zu KV 36 wird KV 36 zum Zentrum bei der Untersuchung der thutmosidischen Belegung des Tals der Könige. Dies ist insofern irritierend, als das Grab eines unter vielen aus dieser Zeit ist. Vielversprechender wäre ein neuer Ansatz, der spezifisch auf analoge Einkammergräber fokussiert unter Berücksichtigung des ausgeschlagenen Innenvolumens (Angaben zur exakten Grabgröße von KV 36 und zu denen der Vergleichsgräber fehlen), Schachttiefe, Position des Grabes im Verhältnis zum Grab des regierenden Königs, Hanglage (Höhe über Talgrund; oft nicht zu ermitteln) und weitere Faktoren, um einerseits eine Kontextualisierung von KV 36 zu erreichen und andererseits zu bestehenden Argumenten weitere Faktoren bestimmen zu können, weshalb ein Grab an einem bestimmten Ort angelegt wurde. ${ }^{11}$ Der bei jedem Grab vorhandene Eintrag „Dekoration“ erweckt den Eindruck, dass thutmosidische Gräber im Tal der Könige, die nicht für Könige angelegt wurden, hätten dekoriert sein können, wofür sich kein einziger Beleg (auch nicht aus Gräbern, die nie geflutet wurden) anführen lässt. Man darf hier umgekehrt argumentieren: Keine Angaben zu Dekoration durch Entdecker und spätere Besucher/Bearbeiter weisen darauf hin, dass keine Dekoration vorhanden war, andernfalls hätte sie Erwähnung gefunden, da das Forschungs-

9 Elisabeth Thomas und Nicholas C. Reeves

10 Dorn, A., Arbeiterhütten im Tal der Könige. Ein Beitrag zur altägyptischen Sozialgeschichte aufgrund von neuem Quellenmaterial aus der Mitte der 20. Dynastie (ca. 1150 v. Chr.), AH 23, Basel 2011, 33 mit Anm. 33.

11 Siehe hierzu den von Lakomy zitierten Artikel von Preys, R., Les tombes non-royales de la Valle des Rois, in: SAK 40, 2011, 315-338. interesse abgesehen von Objektfunden bereits im 19. Jh. und auch später auf der Dokumentation von Texten und Bildern lag. ${ }^{12}$ Die Angaben zur Datierung basierend auf der älteren Literatur, verschiedenen Arbeiten von David Aston und anderen ${ }^{13}$ oder noch nicht publizierten Ergebnissen laufender Grabungen sind meist weit gefasst (z. B. S. 22 KV 27, KV 28: Hatschepsut-Thutmosis IV), was zeigt, dass das Ziel, mittels von Grabinventaren anderer thutmosidischer Gräber aus dem Tal der Könige weitere Argumente für die exaktere Datierung von KV 36 zu finden, aufgrund der aktuellen Publikationslage nicht zu erreichen ist. Das Vorgehen erstaunt aus methodischer Sicht, da alle Vergleichsinventare aus mehr oder weniger gestörten Befunden stammen und diese dem ungestörten Befund gegenübergestellt werden, wobei unter anderem Befunde aus Gräbern mit Mehrfachnutzung vorliegen und diese nur bei Anlagen, die in den letzten ca. 30 Jahren untersucht wurden, differenziert publiziert wurden. Um in Bezug auf die Datierung dieser Gräber weiterzukommen, wäre eine umfassende zeichnerische Dokumentation der Keramik und die anschließende Zuweisung an die Keramikphasen des Neuen Reichs erforderlich. ${ }^{14}$ Angaben in der Rubrik „Parallelfunde zu KV 36“ wie zum Beispiel bei Grab KV 37 (S. 25) „Mehrere Keramikgefäß-Fragmente großer Vorratsgefäße mit einem weißen Überzug/white wash, deren Verbleib unbekannt ist." Den Beschreibungen E. Thomas zufolge sind diese Fragmente mit Gefäßen aus KV 36 und KV 46 vergleichbar. Diese vage Aussage lässt sich für eine genauere Datierung einer Grabanlage nicht verwerten, da die Laufzeit der Gefäße fast die gesamte 18. Dynastie abdeckt und die von E. Thomas als Vergleiche angegebenen Gefäße aus KV 46 (Regierungszeit Amenophis' III.) deutlich jünger sind als die von Lakomy für KV 36 vorgeschlagene Datierung.

In Kapitel II bespricht Lakomy ausführlich und gewinnbringend die Lage (S. 43), die Entdeckungsgeschichte (S. 43-45) und die Bearbeitungsgeschichte des Grabes und der Grabbeigaben (S. 45-49), mit Verweisen, wie detailliert die jeweiligen Studien sind und ob

12 Siehe zum Beispiel Lefébure, E., Notices des hypogées. Les hypogées royaux de Thèbes. MMAF 3/1. Paris 1889.

13 Aston, D., Aston, B. und Ryan, D. P., Pottery from Tombs in the Valley of the Kings, in: CCÉ 6, 11-38; Aston D., Pottery recovered near the tombs of Seti I (KV 17) and Siptah (KV 47) in the Valley of the Kings, AH 24, Basel 2014.

14 Aston, D. A., New Kingdom pottery phases as revealed through well-dated tomb contexts, in: Bietak, M. (ed.), The synchronization of civilisations in the Eastern Mediterraniean in the second millennium B. C. II: Proceedings of the SCIEM 2000 - EuroConference, Haindorf, $2^{\text {nd }}$ of May $-7^{\text {th }}$ of May 2001, Wien 2003, 135-162. Die Keramik aus KV 36 wird von Aston der Phase 2B Amenophis II-Thutmosis IV zugewiesen. 
der Autor sich vor Ort ein Bild des Zustandes des Grabes gemacht hat. Bei der Beschreibung des Grabes (S. 43) geht Lakomy auf Abweichungen der Schachttiefe zur Zeit der Grabentdeckung durch Loret ein, die heute anscheinend deutlich geringer ausfällt. Aus dem Grabplan von KV 36 von Weeks (Abb. 32) geht der Geländeverlauf nicht klar hervor. Zur Zeit der Publikation Lakomys ließ sich deshalb die Schachttiefe nur ungenau bestimmen. Dies wird nun, wenn die Grabungsergebnisse der 2014 durch die Universität Basel erfolgten Nachuntersuchungen vorliegen, welche die Reinigung des Grabes und Ausgrabungen im unmittelbaren Bereich des Schachtmundes beinhalteten, möglich werden. Anhand der Fotos auf Taf. 15 (Abb. 33a-d) lässt sich einerseits feststellen, dass im Westen die Schachtwand noch eine beträchtliche Höhe aufweist und andererseits, dass der Schacht in einer Konglomeratsschicht angelegt wurde und erst ab einer gewissen Tiefe massiver Kalkstein folgt. Diese Konglomeratsschicht, falls die Beobachtungen zur geringen Schachthöhe zutreffen, steht auf den anderen drei Seiten nicht mehr so hoch an. Sie kann bereits bei der Bergung der Objekte abgetragen worden sein, um das Abbrechen von Gesteinsmaterial zu verhindern. Möglich ist aber auch, dass sie bei jüngeren Grabungen im Areal nicht erkannt und abgetragen wurde. Trifft diese Interpretation zu, erübrigt sich auch die Suche nach Gründungsdeposita (s. u.), da das Originalniveau, in welchem sich diese befinden müssten, gemäß den vorliegenden Fotos und Befunden gar nicht mehr vorhanden ist. Dasselbe gilt auch für die Diskussion zu einem postulierten Graboberbau (s.u.). Bei der Grabbeschreibung fehlen dem Rezensenten die Angaben zu den Schachtmassen, dem Durchgang in die Grabkammer sowie zur Fläche und zur Höhe der Grabkammer, die nur aus dem Plan von Weeks hervorgehen bzw. herausgemessen werden müssen sowie eine Verortung des Grabes mit einer Diskussion von Preys Ergebnissen, der das Grab typologisch mit guten Argumenten in die Regierungszeit Amenophis' II. datiert. ${ }^{15}$ Zudem finden die klar sichtbaren Architekturmarken (Abb. 33e) im Türsturzbereich keine Erwähnung. Auf den Seiten 49-51 geht Lakomy der Frage nach, ob sich in Maiherperis Grab „Magische Ziegel“ befanden, da zumindest in einem nicht königlichen, ungefähr gleichzeitigen Grab im Tal der Könige (KV 48) solche Ziegel mit Totenbuchspruch 151 gefunden wurden. Lakomy will die Existenz der Ziegel nicht ausschließen (S. 51) „... verfügt haben könnte.“, da die Wände der Sargkammer nicht systematisch mit dieser Frage abgesucht worden seien. Mit dieser Vermutung unterstellt Lakomy wohl eher unbe-

15 Preys, R., Les tombes non-royales de la Valle des Rois, in: SAK 40, 2011, 324-327. wusst dem Entdecker des Grabes Loret, den Mitarbeitern des Theban Mapping Project, welche das Grab vermessen haben, sowie den University of Basel KV Projektmitarbeitern, die 2014 den Schutt aus dem Grab entfernten, der seit der Entdeckung ins Grab gelangte, das Grab nicht umfassend archäologisch untersucht zu haben. Im Fall des letzt-genannten Projekts ist dies bedauerlich, stellte dieses Lakomy aktuelle Fotos der frisch gereinigten Grabkammer zur Publikation zur Verfügung, auf welchen zu sehen ist, dass sich keine (verdeckten?) Nischen für „Magische Ziegel“" im Grab befinden (Taf. 16-17 mit Abb. 32f-h). Analog präsentiert sich die Diskussion der Gründungsgruben (foundation deposits): Aus dem Tal der Könige sind für Privatgräber keine bekannt und der Verweis auf frühere Grabungstechniken befriedigt nicht, da Ende des 19. und zu Beginn des 20. Jahrhunderts vielerorts systematisch Suchschnitte bzw. flächig bis auf den anstehenden Felsen ausgegraben wurde. Dass sich keine Gründungsgruben in der näheren Umgebung des Schachtmundes befinden, wo diese zu erwarten wären, ist Abb. 33a auf Taf. 15 zu entnehmen. Zweimal (S. 52 und S. 89-95) diskutiert Lakomy das Vorhandensein möglicher Graboberbauten oder anderer Hinweise auf die Lage eines Grabes, deren Existenz er bei Schachtgräbern der Thutmosidenzeit rein strukturell im Prinzip ,,(bislang)“ ausschließt, um mehrfach in der Publikation ihre Existenz wieder in Betracht zu ziehen (S. 93): ${ }^{16}$ „Bedeutet das zwangsläufig, dass es im Tal der Könige für die genannten Grabanlagen von vornherein nicht vorgesehen war, nach der Bestattung ihrer Besitzer generell einen oberirdisch-architektonischen Hinweis auf ihre Lage und ihre Eigentümer anzubringen?“. Diese Frage ist nach Meinung des Rezensenten klar mit Ja zu beantwortet, die Privatgräber der Thutmosidenzeit wurden so angelegt, dass ihre Lage nach dem Einbringen der Bestattung so gut wie möglich verborgen war. Da heute im Tal der Könige fast alle Schächte inklusive KV 36 mit einer Backsteinmauer modern eingefasst und mit einer verschließbaren Klappe versehen wurden, erübrigen sich Nachgrabungen zum Nachweis von oberirdischen Strukturen. Beim Errichten dieser Einfassungen wurden Nachuntersuchungen zu den Schachtöffnungen gemacht und keine Spuren antiker Strukturen gefunden.

Alle von Lakomy angeführten Belege für mögliche Kultorte oder einen Sammelkultort stammen aus der 19. Dynastie oder später, aus einer Zeit, als das Nutzungskonzept des Tals der Könige ein anderes war (s.o.), weshalb auch hier eine Argumentation mit Belegen aus

16 Siehe auch S. 59: "Auch die Frage nach einem hypothetisch anzunehmenden Graboberbau bzw. einer Kultstelle am Grab, steht noch offen." 
der 19. Dynastie für nicht nachgewiesene Praktiken der 18. Dynastie zurückzuweisen sind. Zu einer gänzlich anderen Verortung des als Kultplatz vorgeschlagenen Begriffs $\operatorname{tr}(y) . t$, welcher auf dem aus der Regierungszeit Ramses' II. (19. Dynastie) stammenden Ostrakons JdE 72460 belegt ist und sich auf eine Darstellung in einem Grabinnenraum bezieht, siehe Dorn \& Polis 2016. ${ }^{17}$ Ostraka aus der 19. und 20. Dynastie, die von Arbeitern, welche die Königsgräber errichteten, geschaffen und in ihren eigenen Hütten aufgestellt (nicht als Votivgaben/ex voto zu klassifizieren; anders Lakomy S. 92) und im Rahmen von alltäglichen Gebeten $\mathrm{zu}$ den dargestellten Gottheiten genutzt wurden, können auch nicht als Belege für eine (bereits ab der 18. Dynastie bestehende) Kultstelle im Tal angeführt werden. ${ }^{18}$ Die von Lakomy erwähnte Stele Sen(y)nefers aus der 18. Dynastie lässt sich ebenfalls nicht als Beleg für eine Kultstelle im Tal der Könige anführen, da neben den in der Primärpublikation bereits genannten Argumenten die Giebelszene, ${ }^{19}$ welche die Verehrung eines thutmosidischen Königs durch den Steleninhaber zeigt (iri.n-Vermerk + Personenname), nicht als charakteristisches Motiv einer Stele für den Totenkult gilt.

Die seit Jahren geführte Diskussion nach den Kultstellen und dem Kultvollzug für Beamte aber auch für Mitglieder der Königsfamilie, denen das Privileg (?) zukam, im Tal der Könige bestattet worden zu sein (S. 89-95) wird von Lakomy klar resümiert und mit weiteren Vorschlägen (Kult in der Heimatstadt, Kult in Abydos) bereichert. Bei den neuen wie auch den bestehenden Vorschlägen handelt sich um zahlreiche interessante und in sich meist schlüssige, jedoch nicht beweisbare Hypothesen, die zeigen, dass es im Nachgang zur Trennung von Kultstelle (im Millionenjahrhaus/Totentempel) und Königsgrab (im Tal der Könige verborgen) im Bereich der Elitebestattungen zu unterschiedlichen Lösungen kam. Die „privaten“ Gräber im Tal der Könige setzen das bestehende Modell fort, in räumlicher Nähe zum König bestattet zu sein, so wie dies bis zu den königlichen Pyramidenkomplexen der 17. Dynastie der Fall war, um soziale Bezüge durch geografische Nähe auszudrücken. Andere Formen waren die

17 Dorn, A., Polis, S., A Re-examination of O. Cairo JdE 72460 (= O. Cairo SR 1475). Ending the quest for a $19^{\text {th }}$ Dynasty Queen Tomb in the Valley of the Kings?, in: P. Collombert/D. Lefèbre/S. Polis/J. Winand (Hg.), Aere perennius. Mélanges égyptologiques en l'honneur de Pascal Vernus, OLA 242, Leuven 2016, 129-161.

$18 \mathrm{Zu}$ einer Kultstelle im Tal der Könige für die Göttin Meretseger aus der Ramessidenzeit, siehe Reeves, N. C., The Valley of the Kings Foundation Newsletter 1, Summer 2002, 29.

19 Dorn, A., Grothe-Paulin, E., Die Stele Sen(y)nefers und Grabkegel (Cones) aus dem Tal der Könige, in: GM 222, 2009, 9-12.
Ausrichtung bzw. der Bezug von Gräbern auf das Millionenjahrhaus oder auch auf den Tempel des Gottes Amun von Karnak. Wo der Totenkult für die in undekorierten Gräbern ohne Oberbau (Kultstelle) Bestatteten stattfand, wissen wir schlicht nicht, und jede weitere Hypothese verdeutlich diesen Befund weiter, löst aber das Problem nicht. So darf offen bleiben, ob die Nähe zum Königsgrab mit den Jenseitstexten und dem Kultvollzug für den König im Millionenjahrhaus, an dem die verstorbenen Privatleute $\mathrm{zu}$ partizipieren hofften, in der Vorstellungswelt bereits ausreichte, um den Totenkult für die Privatpersonen zu ersetzen, oder ob es wie im Fall von 'In dazu die physische Präsenz in Form einer Statue brauchte (siehe S. 91-92) oder es allenfalls extrasepulchrale Uschebtidepots in Abydos waren, die diese Funktion übernahmen oder das jedoch nur für einige Beamte belegte und dekorierte Erstgrab in der thebanischen Nekropole außerhalb des Tals der Könige.

Auch der letzte Satz in der Zusammenfassung (S. 306) kreist noch um die Themen Graboberbau, Magische Ziegel und Gründungsgruben, bei denen Lakomy, wie es den Anschein macht, die Resultate archäologischer Arbeit anderer Projekte nicht als ausreichend erachtet (freigelegter Schachtmund: Gründungsgruben; Graboberbau) und zudem nicht zu erkennen scheint, dass mit der modernen Einfassung des Schachtes von KV 36 (siehe Abb. 28) die postulierten Strukturen nicht mehr zugänglich beziehungsweise vorhanden sind und, wie die Ausgrabungen der vergangenen Jahre im Bereich der verschiedensten Schachtgräber zeigten, auch nie vorhanden waren.

Im 3. Kapitel zur Person Maiherperis bespricht Lakomy ausführlich alle belegten Titel (S. 61-83) und geht auf eine mutmaßliche Darstellung Maiherperis in TT 131 ein, die aufgrund nicht vorhandener Namensbeischriften eine nie beweisbare Hypothese bleiben muss, die leider nicht zu einer zusätzlichen Historisierung der Person führt oder zur Datierung des Grabes Maiherperis herangezogen werden kann. Für die Frage nach der Bestimmung der Lebensdaten Maiherperis hat Lakomy eine Liste (S. 75-80) erstellt, in welcher 50 Personen (Grab, Statuen- oder Stelenbesitzer) genannt werden, welche identische Titel oder Titelreihungen wie Maiherperi besitzen. Einige der aufgeführten Titelbelege decken die frühe 18. Dynastie ab, wobei es sich bei den Personen aus dieser Periode um die höchsten Beamten/Vertrauten aus Hatschepsuts Umfeld (die Erzieher Senimen TT 252, Senenmut TT 71/TT 353, vgl. auch Ahmose TT 241) handelt. ${ }^{20}$ Bis auf drei Beispiele

20 Zum Wert älterer Belege zur Entkräftung von Datierungsvorschlägen siehe oben die Kritik an Roehrigs Methodik (S. 68-69). 
(Benja TT 34321 , die Holzstatue des Inipehuisen aus dem Millionenjahrhaus Thutmosis' III., die Statue des Sabastet aus Zagazig) fehlen ausschließliche Belege aus der langen Regierungszeit Thutmosis' III., vielmehr überwiegen die Belege, welche Thutmosis III/Amenophis II-zeitlich oder später datiert sind. In Kombination mit der Auswertung der Funde (,stilistische Analyse des ungenutzten Zedernholzsarges zusammen mit individuell voneinander abweichenden Henkelkrügen und dem (Import-)Glasgefäß ..., dem Totenbuchpapyrus und dem mit dem Thronnamen Königin Hatschepsuts beschrifteten Stück Leinen“) vermutet Lakomy S. 83, dass Maiherperi im „22.-25./(28./29.) Jahr der Alleinherrschaft König Thutmosis’ III.“ bestattet wurde, womit ein ähnliches Datum wie bei Orsenigo (Jahr 20) vorliegt. Keine der im Katalog zu den Objekten der Grabausstattung gegebene Diskussion ergibt ein so enges Datum. Das von Lakomy vorgeschlagene Todesdatum scheint maßgeblich durch die Datierung der anonymen Darstellung eines Wedelträgers im Grab Useramuns (TT 131) beeinflusst zu sein. Der Wedelträger wird von Lakomy mit Maiherperi gleichgesetzt, siehe S. 14: „stelle ich die meines Erachtens berechtigte Vermutung an, diesen (vorsichtig) mit Maiherperi zu identifizieren. Neben dem Schurz, der Kopfbedeckung und der unmittelbaren Nähe zum König entsprach vor allem die Silhouette des dargestellten Wedelträgers der des Maiherperi in den Vignetten seines Totenbuchpapyrus“. Der Grabinhaber Useramun ist außerhalb seines Grabes in einem Jahr 28 das letzte Mal belegt, weshalb seine Grablege im Bereich dieses Regierungsjahres Thutmosis' III. stattgefunden haben könnte (siehe auch S. 250 bei der Diskussion der Datierung des Papyrus).

Der Katalog in Kapitel IV (S. 97-302) stellt das Kernstück der Arbeit dar. Er ist in Bezug auf die Eingangsfrage nach der Datierung der Grabausstattung eine Enttäuschung, da bei allen Objekten beim Datierungsfeld „Mögliche Datierung - NR, frühe 18. Dynastie, Regierungszeit Hatschepsut-Thutmosis' III.“ angegeben wurde. ${ }^{22}$ Dadurch

21 Der Verweis auf die kunsthistorische Datierung des Grabes ins 44.-49. Regierungsjahr Thutmosis' III. durch Guksch, H., Das Grab des Benja, gen. Paheqamen, Theben Nr. 343, Nachtrag zur Publikation des Grabes in AV 7, 1978, in: MDAIK 38, 1982, 195-199, spez. 199 fehlt.

22 Wie verfänglich und widersprüchlich diese konsequent im Katalog verwendet Angabe ist, zeigt sich am mit dem Namen der Königin Hatschepsut (CG 24099) beschrifteten Leinenstück, das in der ganzen Publikation mit ihrer Regierungszeit verbunden wird. Hier ist die Angabe Thutmosis III fehl am Platz, außer die Angabe wäre als Jahr 1-20 der gemeinsamen Herrschaft zu lesen, was dann im Wiederspruch zur Grabdatierung Jahr 22-29 Thutmosis' III. stehen würde. wird bei jedem Objekt eine andere Datierung per se ausgeschossen - außer man gewichtet das abschwächende möglich anders. Zudem werden durch die in der Publikation wiederholt verwendete Aussage (siehe z. B. S. 14; Zitat hier S. 262) „auf der Basis der Kontext bezogenen Auswertung des gesamten Grabinventars" auf zirkuläre, nicht diskursive Weise andere insbesondere jüngere Datierungsansätze für ein Objekt ausgeschlossen. Es fehlt eine Diskussion zur Belegspanne von Objekten (s. o. die Kritik an Roehrig) wie zum Beispiel dem schwarzgrundigen Sarg, dem Kanopenkasten (s. u.) oder der Gesichtsmaske und weiteren anderen Objekten. Stattdessen finden sich zwei verschiedene idiosynkratische Kategorien zur Diskussion von Vergleichsobjekten: „Kategorie I: Vergleichsobjekte (bedingt vergleichbar)“ und „Kategorie II: Vergleichsobjekte (direkt vergleichbar)“, die so bei archäologischen Objektvorlagen nicht gebräuchlich sind. Der Autor klärt insbesondere bei direkt vergleichbaren Objekten den Wiederspruch zu seiner Datierung „Mögliche Datierung - NR, frühe 18. Dynastie, Regierungszeit Hatschepsut-Thutmosis’ III.“ nicht auf, der bei der Erwähnung von deutlich jüngeren Vergleichsbeispielen entsteht, wie zum Beispiel bei den großen Vorratsgefäßen (s. bereits o.), wo auf ein Gefäß (1/43) aus dem Grab Tjannunis (TT 74; Zeit Thutmosis' IV.) oder sogar aus KV 55 (späte 18. Dynastie) verwiesen wird. ${ }^{23}$

Die datierende Relevanz der gegebenen Vergleiche ist für den Leser nur mit einem gewissen Aufwand ersichtlich, da meist keine Daten zu den Vergleichsstücken angegeben sind, sondern mehrheitlich Grabnummern aus Theben (TT), so dass sich der Leser selbst die Datierung der einzelnen Vergleiche (teilweise in der Publikation selbst zu finden) beschaffen muss. Zudem wird auf Vergleichsstücke in Museen verwiesen, zu deren Herkunft keine archäologischen Kontexte bekannt und deren Inhaber teilweise nicht weiter historisierbar sind. Der Fokus bei den Vergleichen

23 Zur Belegspanne dieser Gefässe siehe Aston, D. A., Pottery of the Egyptian New Kingdom: A Study. Eighteenth dynasty Nile clay storage jars from the Valley of the Kings (in Druck für eine Festschrift). In diesem Artikel, der eine Typologie der Gefäße inklusive ihrer Datierung enthält, sind die drei im Grab verbliebenen großen weißgrundigen Vorratsgefäße in Zeichnung enthalten (vgl. Abb. 33i). Von Aston werden die Gefäße in die Regierungszeit Thutmosis' IV. datiert, wobei das ganz erhaltene Profil von Gefäß KV 36-3 "similar to the jars from the reign of Tutmosis III” ist, jedoch einen runderen Rand hat. Das Gefäß KV 36-4 weist einen deutlich längeren und fast geraden Hals auf sowie einen umlaufenden Wulst (collar) im Bereich des Halsansatzes, wodurch es sich deutlich von den Thutmosis III-zeitlichen Gefäßen unterscheidet und abgesehen von zwei umlaufenden Rillen im Halsbereich mit einem Gefäss (1/43) aus dem Grab des Tjannuni (Bestattung zur Zeit Thutmosis IV.) vergleichbar ist. 
liegt auf älteren Vergleichsstücken, die das eingangs in der Publikation postulierte Begräbnisdatum (u.a. S. 14-15) stützen, ohne in den meisten Fällen jüngere Vergleichsbeispiele gleichwertig zu diskutieren. Unter „bedingt vergleichbar" sind jüngere Objekte aufgeführt, die für die chronologische Diskussion nicht weiter berücksichtigt werden. Da ihre exakte Datierung nicht vermerkt ist und Angaben zur Belegspanne der jeweiligen Objektgattung fehlen, wird es dem Leser erschwert, auf diese für eine kritische Datierungsdiskussion zurückzugreifen, so dass sich der Wert der Nennung von „bedingt vergleichbaren“ Objekten auf einen rein phänomenologischen beschränkt. Die Datierungsproblematik zeigt sich exemplarisch an der Diskussion der Keramik, welche die „aussagekräftigsten Kriterien für eine exakte Datierung“ (S. 166) liefert. Sie basiert auf elf Henkelkrügen und drei (kleinen) Amphoren (CG 24021-24023) mit meist bichromer Bemalung (siehe Exkurs V S. 166-168). ${ }^{24}$ Sie ist methodisch nicht haltbar (siehe auch die nicht weiter diskutierten Verweise auf im Flachbild dargestellte Gefäße) und lässt außer Acht, dass generell das jüngste Objekt in einem Konvolut d.h. in der gesamten Grabausstattung inklusive Keramik datierend ist. Lakomy fokussiert bei seiner Analyse auf frühe Belege für Henkelkrüge(Ahmose/Amenhotep I.), ohne deren Laufzeit zu bestimmen und resümiert im Folgenden auf S. 167 „Damit bestätigen diese Krüge - und alle anderen Vergleichsobjekte im folgenden Katalogteil - die These, dass die unten aufgeführten Henkelkrüge und die (kleinen) Amphoren aus KV 36 ihre absolut datierenden Vergleichsstücke vor allem in der gemeinsamen Regierungszeit Königin Hatschepsuts und König Thutmosis' III. bzw. in der unmittelbar anschließenden Alleinherrschaft König Thutmosis’ III. finden.“ Dass der Befund nicht so eindeutig ist wie dargestellt, illustrieren die Angaben in einer der in Anmerkung 344 zitierten Literaturangabe. ${ }^{25}$ Aston diskutiert mit Verweisen auf weitere Literatur die Belegspanne für vergleichbare Henkelkrüge, die bis in die

24 Den Kommentar „doppelter Irrtum“ zu Aston 2009 (S. 167 Anm. 345) hätte sich der Autor sparen können, da die Angaben von Aston korrekt sind. Aston diskutiert an dieser Stelle Objekte aus dem Grab Amenophis' II. (KV 35), welche im selben Catalogue Général Band publiziert sind wie die Grabausstattung Maiherperis unter Angabe korrekter Tafel- und Seitenverweise. Zudem scheint der Autor „potmarked“ mit hieratischen (Tinten-),,Aufschriften“ bzw. „Vermerken“ zu verwechseln, wie sie sich auf Gefäßen Maiherperis finden. Potmarks bezeichnen jedoch vor dem Brand angebrachte Markierungen, bei den nach dem Brand angebrachten Markierungen handelt es sich um „workmen's marks“.

25 Aston D., Pottery recovered near the tombs of Seti I (KV 17) and Siptah (KV 47) in the Valley of the Kings, AH 24, Basel 2014, 23.
Regierungszeit Thutmosis' IV. reicht. ${ }^{26}$ Eine Verortung der Henkelkrüge in dieser Zeitspanne bleibt Lakomy schuldig, hingegen ist in Abb. 275 ein Henkelkrug abgebildet, der ein Siegel Thutmosis' III. trägt. Dieser frontal fotografierte Henkelkrug unterscheidet sich typologisch von denen Maiherperis durch seinen breiten Hals und einen senkrecht nach unten führenden Henkel mit größerer Henkelöffnung. Wie weit ein kantiger Gefäßumbruch im Bereich vom maximalen Durchmesser vorliegt, lässt sich ab dem Foto nicht abschließend sagen. Vergleiche von diesem Gefäß mit den Gefäßen aus KV 36 werden durch die unsystematische, nicht frontale fotografische Wiedergabe der Gefäße Maiherperis erschwert (s. Taf. 82-97). Alle Henkelgefäße aus KV 36 weisen schmalere Hälse auf und ihre Henkel sind rund, ziehen bei der Verbindung mit dem Gefäßkörper deutlich ein (am wenigsten CG 24019).

Bei der Datierung von zwei der drei kleinen Amphoren (CG 24021) mit horizontalen Henkeln verweist Lakomy (S. 181 mit Anm. 430) auf ein Vergleichsstück aus einem datierbaren Fundkontext mit der nicht korrekten Angabe „Provenienz: Unbekannt“. Die kleine Amphore stammt aus Grab CC41, pit 3, chamber B, welches ins frühe Neue Reich datiert und in der Zeit nach dem 7. Regierungsjahr Hatschepsuts bei der Anlage des Aufweges zu ihrem Millionenjahrhaus versiegelt wurde. ${ }^{27}$ Das Gefäß weist typologisch ebenfalls deutliche Unterschiede auf: breiterer Hals, schlankere, fast senkrecht stehende Henkel und deutlicher Gefäßumbruch beim maximalen Durchmesser, zudem ist die Bemalung gänzlich anders. Bei beiden Gefäßtypen, Henkelkrügen und kleinen Amphoren, welche als die den Befund maßgeblich datierenden Objekte diskutiert wurden, fehlt deren klare Verortung innerhalb der Belegspanne der jeweiligen Gefäßtypen, weshalb die so sichere Datierung der Grabausstattung von KV 36 bei weitem nicht so eindeutig ist, wie vom Verfasser postuliert und gemäß dem Rezensenten nicht auf das enge Zeitfenster 22.-29. Regierungsjahr Thutmosis' III. festgelegt werden kann, wofür der Autor auch klare Argumente schuldig bleibt.

Die beiden Kapitel (Exkurs II: Fertigungszeitpunkt und Wert ausgesuchter Beigaben aus KV 36, S. 55-58 und IV.5 Die nachträglich zu ergänzenden Beigaben, S. 264266) zeigen auf, wie breit die zeitliche Streuung von Objekten einer Grabausstattung sein kann. Hieran lassen sich weitere Diskussionen zur Zusammensetzung von Grabaus-

$26 \mathrm{Zu}$ weiteren Belegen für die Verwendung von bichromem Dekor auf anderen Gefässformen siehe Aston, D. A., Amphorae in New Kingdom Egypt, in: Ägypten und Levante 14, 2004, 184.

27 Siehe die Angaben bei Roehrig C. H., Dreyfus, R., Keller C. A., Hatshepsut. From Queen to Pharaoh, New York 2005, 232 und insbesondere 233 . 
stattungen, Entsorgung von Altstücken aus königlichen Werkstätten und aus Familienbesitz sowie jüngeren und älteren persönlichen Gegenständen anschließen, was im Rahmen einer differenzierten Datierungsdiskussion, wie sie von Lakomy angestoßen wurde, durchaus zu weiteren Erkenntnisse zu Bestattungspraktiken von Mitgliedern der Elite des Neuen Reiches führen dürfte.

Für die Datierung des Grabensembles schlage ich ein in einem ersten Schritt nicht auf einzelne Objekte fokussierendes Vorgehen vor: Als Terminus postquem non lässt sich zum Beispiel die Bestattung von Kha und Merit TT 8 ansetzen, deren Objekte eine zeitliche Streuung von Amenophis II-Amenophs III aufweisen. Als Terminus antequem non dient die Kartusche Hatschepsuts auf dem Leinenstück CG 24099 aus dem Grab KV 36. Lässt sich nun ein Grabensemble finden, das weiter zur Eingrenzung des Grabensembles Maiherperis herangezogen werden kann, wie zum Beispiel die Bestattungen der „Three foreign wives“28? Wie verhält sich dieses zur Grabausstattung Maiherperis, lassen sich die chronologischen Ausreißer bestimmen, welches Objekt ist nun als jüngstes, datierendes anzusetzen? Auf diese Weise dürften sich Zirkelschlüsse vermeiden lassen und wie von Lakomy zu Recht gefordert, kontextualisierte Ergebnisse gewinnen, ohne beim abschließenden Datierungsvorschlag auf eine so exakt wie mögliche Datierung der jüngsten Objekte zu verzichten.

Am Katalog ist die nicht maßstäbliche Objektvorlage (Zeichnungen und Fotos) zu bemängeln, was auf einen kunsthistorisch ästhetischen Zugang des Autors zum Material hinweist. Ausnahmen stellen u. a. die Zeichnung zum Sarg CG 24100, zum Sargdeckel CG 24101, CG 24002 (Abb. 100) zum ungenutzten Holzsarg CG 24003 und ein Foto von einer Geflügelbeigabe mit direkt auf das Artefakt gelegtem Maßstab (Abb. 415; 417) dar. Die Zeichnungen genügen Ansprüchen an archäologische Objektzeichnungen nicht (siehe zum Beispiel die Zeichenformen der zeichnerisch anspruchsvollen Unterseite des Herzskarabäus Abb. 566 und 567) und sind eher als impressionistisch zu klassifizieren: siehe zum Beispiel die Kopfpartie des äußeren anthropoiden Holzsarges (CG 24002) in Abb. 93 im Vergleich mit Abb. 96-99 (Taf. 28-29) insbesondere die schwarzen Augenbrauen und Augeneinfassungen, der Verlauf des linken oberen Augenstrichs im Nasenbereich, fehlende Angaben der zwei Dübel (Wange rechts, Mund-

28 Lilyquist, C., The Tomb of three foreign wives of Tuthmosis III, New York 2003 mit einer nicht allzu großen Anzahl an vergleichbaren Objekten, was einerseits statusbedingt ist und andererseits an der Beraubung von wertvolleren Objekten im Grab Maiherperis liegt. winkel links), die Umsetzung des Mundes oder des Nasenbeins.

Als zusätzliche Visualisierung der Position der Objekte der Grabausstattung gibt Lakomy abgesehen vom Plan (Abb. 35, siehe hierzu bereits oben) auch eine perspektivische Zeichnung (Abb. 36), in welcher der Grabschacht fälschlicherweise an der Nordwand statt an der Ostwand der Grabkammer wiedergegeben wurde.

Kurzkommentare zu einzelnen Objekten:

Zum Sarg CG 24001 merkt Lakomy S. 103 an, dass er aus 30 Einzelteilen besteht, die im Grab zusammengefügt worden sind. Dies hätte zur Folge, dass der Bitumenüberzug (,Holzplanken ... und danach innen und außen vollständig mit Bitumen überzogen“) im Grab erfolgt wäre und anschließend auch dort die Goldfolien (Figuren, Inschriften) angebracht worden wären. Diese Annahme halte ich für nicht korrekt. Der Sarg wurde ganz angeliefert, sei es auf einem Transportschlitten, sei es an durch die Metallringe geführte Tragestangen getragen, und anschließend an Seilen, die durch die Metallringe geführt wurden, ins Grab hinuntergelassen.

Die umfassende fotografische und zeichnerische Dokumentation des Kanopenkastens (S. 146-155) CG 24005 zeigt, dass sich auf der Höhe der Metallringe beidseits horizontale Beschädigungen finden, die von den durch die Ringe geführten Tragestangen herrühren, was mit dem Befund nicht vorhandener Abnutzungsspuren an den Kufen des Kasten korrespondiert. Auf Beobachtungen, die auf Nutzungsspuren etc. verweisen, wurde bei den Beschreibungen weitestgehend verzichtet, da die Objektvorlage vielmehr auf das Objekt und die Vergleichsstücke fokussiert. Die Qualität der Dokumentation ermöglicht es jedoch, „object histories“ zu erstellen. Der weißgrundige (nicht schwarze) und im Gegensatz zum Kasten Maiherperis undekorierte Kanopenkasten der Hatnefer weist unter den Kufen zwei stabilisierende Querleisten auf und eignet sich als Vergleichsbeispiel kaum. Zudem ist die Hohlkehle nur wenig ausgeformt. Die einzige Übereinstimmung liegt in der quadratischen (?) Form des Kastens, ein Merkmal, das sich auch bei Kanopenkästen des Mittleren Reiches findet. Was Lakomy bewog, den Kanopenkasten (vgl. Abb. 199 mit 215) und die Mumienmaske (vgl. Abb. 194 mit 195) Hatnefers als „direkt vergleichend“ mit den Objekten Maiherperis anzusehen, erschließt sich dem Rezensenten nicht. ${ }^{29}$ Bei den anderen Vergleichsbeispielen zum Kanopenkasten bestehen ebenfalls zahlreiche Unterschiede. Alle weisen einen stärker gewölbten Deckel auf (die Deckelform des Kastens ist in der Beschreibung nicht erwähnt und in den Zeichnungen und auf den Fotos außer evtl. in Abb. 205 nicht zu erkennen), der eine Kasten ist quadratisch, der andere nicht und bei der Dekoration fällt auf, dass Maiherperis Kasten als einziger ein Palastfassadendekor auf den Seiten aufweist und dass die Göttinnen je auf einem Goldthron/Nebu-Zeichen stehen, wodurch formal deutliche Unterschiede bestehen. Der als direkter Vergleich aufgeführte Kasten der Ruju ist 20 Jahre jünger als das für die Bestattung Maiherperis angesetzte Bestattungsdatum.

Zum Arm- oder Fußreif aus Pflanzenfasern CG 24062 ter (S. 213214): Bei diesem Objekt, das 1902 bei der Mumienauswicklung unter dem Kopf der Mumie lag, dürfte es sich vielmehr um eine Art Hypokephalos handeln. Je nach Handumfang konnte sich Maiherperi zu

29 So auch Roehrig, C. H., The tomb of Maiherperi in the Valley of the Kings, in: Roehrig C. H., Dreyfus, R., Keller C. A., Hatshepsut. From Queen to Pharaoh, New York 2005, 70. 
Lebzeiten den Pflanzenfaserring mit einem Innendurchmesser von 6,5 cm nicht über die Hand ziehen, ein Tragen am Fußgelenk schließe ich aus und der Vorschlag, dass Maiherperi den Reif nach der Balsamierung am Körper trug, da er Verfärbungen aufweist, scheint dem Rezensenten etwas weit hergeholt zu sein.

Das Totenbuch Maiherperis wird an verschiedenen Stellen in der Publikation besprochen: im Katalog (S. 248-252), als eigenes Kapitel IV.6.1-6.5 (S. 266-269), gefolgt von der Übersetzung und unter IV.6.7 (S. 300-302) findet sich „Eine Auswahl datierender Vergleichsobjekte“. Dem Rezensenten fehlt eine vergleichende Analyse der Auswahl des Spruchgutes, stattdessen finden sich neben der Diskussion einiger Totenbuchpapyri aus der 18. Dynastie, u. a. Papyrus $\mathrm{Nu}$ (pBM EA 10477) ${ }^{30}$, Verweise auf verschiedene Leinenfragmente mit Ähnlichkeiten in Bezug auf „Schriftzug“ (Duktus) und „paläographische Gestaltung“, die sich „ganz unabhängig von dem Inhalt des religiösen Spruchgutes mit dem Totenbuchpapyrus des Maiherperi vergleichen“ lassen (S. 300). Hier wäre eine tabellarische oder andere Form der Visualisierung wünschenswert, um die Vergleichbarkeit von Zeichen und Duktus, auf welcher Schlussfolgerungen für die Datierung basieren, nachvollziehen zu können. Bei der kunsthistorisch ikonographischen Verortung des Papyrus, bei welcher Lakomy auch auf die älteren Diskussionen zum Schurz Maiherperis eingeht, verweist er auch auf ein unpubliziertes Sandsteinfragment, das er in einer eigenen Umzeichnung (Abb. 565) als Vergleichsobjekt anführt. Problematisch ist hier die Abhängigkeit des Lesers von einer Umzeichnung eines Sandsteinobjekts (versenktes Relief) zum Vergleich mit Fotos des Papyrus (Malerei). Gemäß den Angaben der Kuratoren des Ägyptischen Museums in Kairo stammt das Sandsteinfragment aus Theben-West. Als Herkunftsort wird die „Kapelle/der Tempel (?) des Sohnes König Thutmosis’ I., Wadjmose“ genannt, was ohne weitere Angaben zur Person des Suemniut und zum exakten archäologischen Kontext und zum Verhältnis zum Bau des Wadjmose keine Datierungsangabe darstellt. So verweist Lakomy auf ikonographische Übereinstimmungen zum Totenbuchpapyrus des Maiherperi, „die sich sehr gut in die frühe Thutmosidenzeit datieren lassen“ unter Angabe von Russmanns „guter Einführung ... zur Kunst des frühen Neuen Reiches“. ${ }^{31}$

Eine gewisse Irritation löste beim Rezensenten einerseits die Bezeichnung „Grabschatz“ aus (S. 13-14 Einleitung; S. 305 Zusammenfassung), handelt es sich doch schlicht und einfach um eine Grabausstattung eines Beamten aus dem näheren Umfeld des Königs - die zudem um zahlreiche wertvollere Gegenstände beraubt wurde - und andererseits die Nähe von Lakomy zu seinem Forschungsgegenstand, wobei der Eindruck entstand, dass sich Lakomy zum Fürsprecher von Maiherperi macht, der in der Forschung gemäß dem Autor (S. 13) „nicht die breite wissenschaftliche Beachtung erfahren“ hat, „die er zweifellos verdient hat“. Dieses Versäumnis holt der Autor mit der reich bebilderten Publikation nach, welche die weitere Beschäftigung mit den qualitätvollen Objekten der Grabausstattung Maiherperis erleichtert und auf einem neuen Niveau möglich macht. Der enzyklopädische Zugang mit der unkommentierten Auflistung einer großen Fülle an Literatur bei den jeweiligen Objekteinträgen illustriert jedoch durchaus das Gegenteil (vgl. auch das Literaturverzeichnis S. 311-331), dass es sich bei der fast gänzlich erhaltenen Grabausstattung Maiherperis um einen außergewöhnlichen Befund handelt, der durchaus rezipiert wurde.

Beide Publikationen zeigen auf unterschiedliche Weise die Bedeutung der Grabausstattung für die ägyptologische Forschung auf, wobei diese jedoch in keinem Verhältnis zur inexistenten Präsenz Maiherperis in der historischen Dokumentation steht, in die ein Beamter nicht automatisch Eingang findet, auch wenn durch das Amt des Wedelträgers seine unmittelbare Nähe zum König bezeugt ist. So mag gerade das Privileg des undekorierten Grabes im Tal der Könige dazu geführt haben, dass zu Maiherperis Leben sonst nichts bekannt ist, da er kein dekoriertes Felsgrab besaß, in welchem besondere Ereignisse oder Angaben zu weiteren Familienmitgliedern hätten vermerkt sein können.

Die deutliche Kritik an beiden Publikationen insbesondere an den methodologisch nicht haltbaren Datierungsansätzen soll den Wert beider Publikationen in keiner Weise schmälern. Beide Autoren tragen mit ihren Publikationen dazu bei, dass die Grabausstattung Maiherperis auf einer völlig neuen Datenbasis untersucht werden kann: Einerseits ist der Grabbefund mit der Position der Objekte (Orsenigo) nun gesichert und andererseits sind die einzelnen Objekte auf bestmögliche Weise beschrieben und in Farbfotos verfügbar, was durch die Kritik des Rezensenten an der Wiedergabe der Objekte nicht geschmälert werden soll, da er einerseits aus eigener Erfahrung weiß, was es heißt, Objekte vor Ort im Museum zu dokumentieren und andererseits Lakomy abschließend (S. 306) darauf verweist, dass es ihm in Abweichung zu seinem Dokumentationskonzept nicht möglich war, „das Grabinventar Maiherperis erschöpfend zu dokumentieren“ ... und „kein Keramikgefäß aus KV 36 maßstabgetreu gezeichnet“" werden konnte.
30 Dieser wird in der Einleitung der Publikation von W. V. Davies in die Mitte der 18. Dynastie datiert, vom Lapp (S. 18) mit Verweis auf Milde und auf die Vignetten ,in the (early) Eighteenth Dynasty“. Auf S. 17 findet sich eine Tabelle mit bestehenden Datumsvorschlägen, die von Hatschepsut/Thutmosis III./1. Hälfte 18. Dynastie bis Amenophis III. reichen und die Datierungsunsicherheit für „eine aussagekräftige Parallele zeigen. Siehe Lapp, G., The Papyrus of Nu (BM EA 10477), London 1997.

31 Russmann, E. R., Art in Transition. The Rise of the Eighteenth Dynasty and the Emergence of the Thutmoside Style in Sculpture and Relief, in: Roehrig et al. op cit., 23-48. 